\title{
Malignant struma ovarii harboring a unique NRAS mutation: case report and review of the literature
}

\author{
Carlo Gobitti, ${ }^{1}$ Alessandro Sindoni, ${ }^{2}$ Chiara Bampo, ${ }^{2}$ Tanja Baresic, ${ }^{2}$ Giorgio Giorda, ${ }^{3}$ \\ Lara Alessandrini, ${ }^{4}$ Vincenzo Canzonieri, ${ }^{4}$ Giovanni Franchin, ${ }^{1}$ Eugenio Borsatti ${ }^{2}$
}

\begin{abstract}
${ }^{1}$ Division of Radiotherapy, Centro di Riferimento Oncologico, IRCCS-National Cancer Institute, Aviano, PN Italy; ${ }^{2}$ Unit of Nuclear Medicine, Centro di Riferimento Oncologico, IRCCS-National Cancer Institute, Aviano, PN Italy; ${ }^{3}$ Gynecological Oncology Unit, Centro di Riferimento Oncologico, IRCCS-National Cancer Institute, Aviano, Italy; ${ }^{4}$ Pathology Unit, Centro di Riferimento Oncologico, IRCCS-National Cancer Institute, Aviano, Italy
\end{abstract}

\begin{abstract}
Struma ovarii (SO), a rare tumor containing at least $50 \%$ of thyroid tissue, represents approximately $5 \%$ of all ovarian teratomas; its malignant transformation rate is reported to occur in up to $10 \%$ of cases and metastases occur in about 5-6\% of them. We describe a 36-year old woman who underwent laparoscopic left annessectomy two years earlier because of an ovarian cyst. Follow-up imaging revealed a right adnexal mass, ascitis and peritoneal nodes that were diagnosed as comprising a malignant SO with peritoneal secondary localizations at histopathology performed after intervention. Restaging with ${ }^{18}$ F-FDG-PET/CT scan, abdominal CT and ultrasonography showed abnormalities in the perihepatic region and presacral space and left hypochondrium localizations. The patient underwent thyroidectomy, hepatic nodulectomy and cytoreductive peritonectomy: histopathological examination did not show any malignant disease in the thyroid and confirmed the presence of peritoneal localizations due to malignant SO; molecular analysis detected NRAS Q61K mutation in exon 3, whereas no mutations were identified on the BRAF gene. The patient underwent radioiodine treatment: serum $\mathrm{Tg}$ was decreased at first follow-up after three months of ${ }^{131}$ I-therapy. We believe that our case raises some interesting considerations. First, pathologists should be aware of this entity and should check for the presence of point mutations suggesting an aggressive disease behavior, which could be beneficial for an optimal therapeutic approach. Second, although most of the knowledge in this field comes from case reports, efforts should be made to standardize the management of patients affected by malignant $\mathrm{SO}$, including use of practice guidelines.
\end{abstract}

Key words: Malignant struma ovarii, Papillary thyroid carcinoma, I-131, Radioiodine, Treatment

Address for correspondence:

Carlo Gobitti, MD. Unit of Radiotherapy, CRO Aviano National

Cancer Institute, via Gallini 2, 33081 Aviano (PN), Italy;

E-mail: cgobitti@cro.it

Received:16-09-2017, Accepted:20-09-2017

\section{INTRODUCTION}

Germ cell tumors, which are reported to comprise $15-20 \%$ of all ovarian cancers, are mostly mature cystic teratomas. ${ }^{1}$ Struma ovarii (SO) is a rare tumor 
classified as a mature ovarian teratoma which contains at least $50 \%$ of thyroid tissue and represents approximately $5 \%$ of all ovarian teratomas. ${ }^{1}$ It was first described by Buttlin in 1888 and the demonstration of the presence of thyroid tissue was performed by Pick 15 years later. ${ }^{2}$ Malignant transformation of SO with areas of well-differentiated carcinoma (mainly papillary histotype) is reported in $5-10 \%$ of all cases; $;{ }^{3,4}$ it is metastatic in $5-6 \%$ of them. ${ }^{5}$ It has mainly been reported in the fifth and sixth decades of life as an unilateral adnexal mass, often left sided. ${ }^{6-8}$ Up to $92 \%$ of patients are euthyroid. ${ }^{8-10}$ Hyperthyroidism is found in the remaining patients (about $8 \%$ ), ${ }^{5}$ but even in this case patients may not shows signs or symptoms of thyrotoxicosis and may only complain of tumorassociated symptoms such as pelvic pain. Because of the rarity of malignant $\mathrm{SO}$, uncertainties can arise about the management of these cases. There are few data in the literature concerning treatments and follow-up approaches in this setting: in particular, after surgery there are no clear guidelines recommending either surveillance alone or adjuvant treatment. ${ }^{11}$ We report the case of a woman with a pelvic mass that proved to be a SO containing a follicular variant of papillary thyroid carcinoma. At the time of abdominal tumor debulking we performed thyroidectomy, followed by radioiodine and levothyroxine suppressive therapy.

\section{CASE REPORT}

A 36-year old woman was referred to our Institution for medical advice. Her past history disclosed laparoscopic left annessectomy two years earlier because of an ovarian cyst. Follow-up controls revealed the presence of a pelvic mass at palpation and pelvic ultrasound (US) showed a right adnexal mass, ascitis and peritoneal nodes. The patient was scheduled for surgery. She underwent hysterectomy and right annessectomy, appendicectomy and omentectomy plus excision of multiple peritoneal nodules. The ovarian mass (10 $\mathrm{cm}$ in maximum diameter) was solid and cystic with cysts containing mucoid material. The solid area had a characteristic red-brown cut surface. Pathological examination revealed the presence of a malignant SO, follicular variant of papillary thyroid carcinoma, with multiple uterine, parametrial, peritoneal, pararectal, periappendicular and perivescical secondary localizations.

The patient was then referred to the multidisciplinary thyroid tumor board of our Institute. Workup for thyroglobulin ( $\mathrm{Tg}$ ) disclosed elevated serum values $(1000 \mathrm{ng} / \mathrm{ml})$ suspicious for persistence of disease; serum TSH, thyroid autoantibodies, FT3 and FT4 were in the normal range and consistent with a euthyroid status. Excluding two cystic areas of $4 \mathrm{~mm}$ in maximum diameter in the right lobe, US of the thyroid showed no other abnormalities. ${ }^{18} \mathrm{~F}-\mathrm{FDG}-\mathrm{PET} / \mathrm{CT}$ scan (Figure 1) performed for disease staging revealed the presence of areas of abnormal radiopharmaceutical uptake in the liver (VII segment), presacral area and left hypochondrium (consistent with a peritoneal secondary lesion); moreover, non-specific diffuse increased radiopharmaceutical uptake was found in the spleen. Abdominal US showed peri-hepatic and intra-abdominal pelvic nodes (36 $\mathrm{mm}$ in max diameter), suspicious for peritoneal secondary localization. Abdominal CT confirmed all PET and US findings.

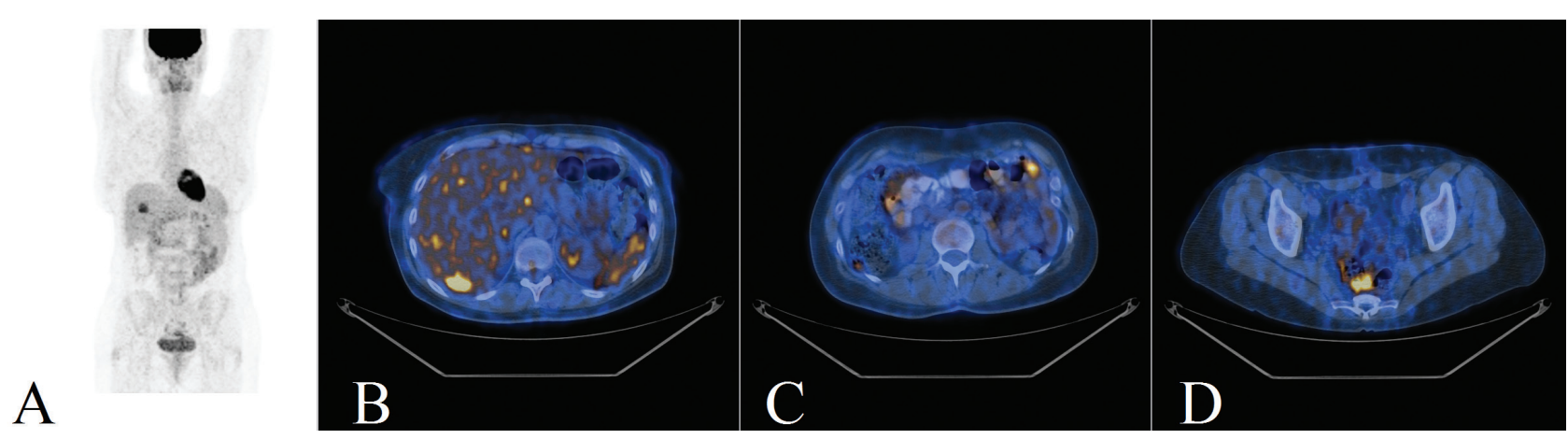

Figure 1. ${ }^{18}$ F-fluorodeoxyglucose PET images. (A) MIP image. Perihepatic (B), left hypocondrium (C) and pelvic (D) peritoneal localizations of monophasic ovarian teratoma (so-called malignant struma ovarii). 
The combined imaging results pointed to the need for another abdominal exploration. The resultant findings were discussed with the patient and the decision for total thyroidectomy and nodular excision of peritoneal masses was made. The patient underwent thyroidectomy, hepatic nodulectomy and cytoreductive peritonectomy, but a radical surgical procedure was not feasible and residual abdominal macroscopic disease remained: histopathological examination did not show any malignant disease in the thyroid and confirmed the presence of peritoneal and hepatic localizations from malignant SO (Figures 2A and B); molecular analysis detected $\mathrm{N} R A S$ Q61K mutation in exon 3, whereas no mutations were identified on the BRAF gene. The patient started levothyroxine therapy at the daily dosage of $100 \mathrm{mcg}$. One month after surgery serum TSH was $0.19 \mathrm{UI} / \mathrm{ml}$ and serum $\mathrm{Tg}$ was 858 $\mathrm{mg} / \mathrm{ml}$. The patient was scheduled for ${ }^{131} \mathrm{I}$-therapy with the following protocol, to avoid progression of pelvic disease due to hormone withdrawal: recombinant human TSH (rhTSH, 0.9 mg i.m.) administration on days 1 and 2 and a therapeutic dose of $5550 \mathrm{MBq}$ of ${ }^{131} \mathrm{I}$ on day 3 . On the day of ${ }^{131} \mathrm{I}$ administration, serum TSH and serum Tg were $155 \mathrm{UI} / \mathrm{ml}$ and 2140 $\mathrm{ng} / \mathrm{ml}$, respectively. The treatment was well-tolerated and the patient experienced no adverse effects. Posttherapy whole-body scan performed 7 days after radiopharmaceutical administration (Figure $3 \mathrm{~A}$ and

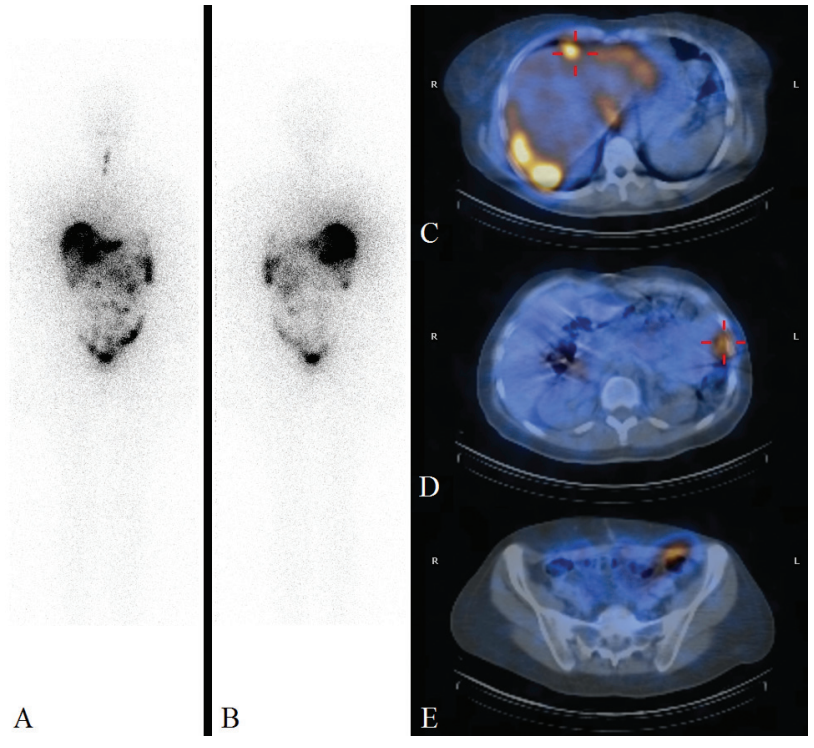

Figure 3. Post-therapy whole-body scan (A, anterior view; B, posterior view) performed 7 days after the therapeutic effect of $5550 \mathrm{MBq}{ }^{131}$ I showed the presence of perihepatic (C), left hypochondrium (D) and lower abdominal areas of abnormal uptake (E).

B) showed the presence of perihepatic (Figure 3C), left hypochondrium (Figure 3D) and lower abdominal areas of abnormal ${ }^{131} \mathrm{I}$ uptake (Figure 3E). Serum Tg was decreased at first follow-up 3 months following the ${ }^{131} \mathrm{I}$-therapy $(483.5 \mathrm{ng} / \mathrm{ml}$; TSH $0.02 \mu \mathrm{UI} / \mathrm{ml})$. The

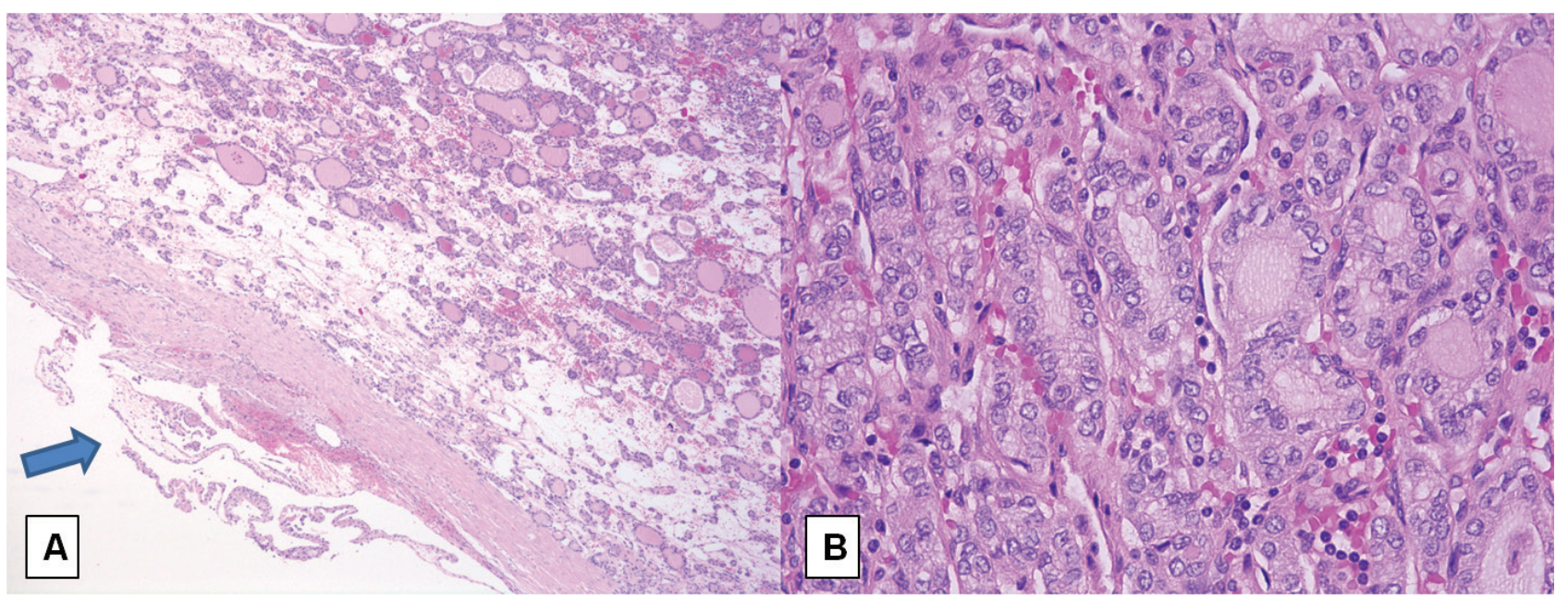

Figure 2. (A) Peritoneal localization of papillary thyroid carcinoma, follicular variant, arising in malignant struma ovarii. An outer mesothelial lining (arrow) is seen (hematoxylin and eosin [H \& E] staining; magnification $\times 50$ ). (B) Higher magnification of Figure A. The neoplasm was composed of small to medium sized follicles containing colloid. The follicular epithelium showed clear, sometimes overlapping nuclei, with gooves and pseudoinclusions. (H\&E staining; magnification $\times 400$ ). 
patient was then enrolled for further administrations of ${ }^{131} \mathrm{I}$ at therapeutic activities.

\section{DISCUSSION}

$\mathrm{SO}$ is a rare tumor classified as a mature ovarian teratoma which contains at least $50 \%$ of thyroid tissue; it represents approximately $5 \%$ of all ovarian teratomas. ${ }^{1}$ Malignant SO is diagnosed when the histopathologic criteria of thyroid carcinoma are found. After surgical resection of malignant SO, the therapeutic approach depends on the extent of the primary lesion and disease staging. In fact, optimal treatment of malignant $\mathrm{SO}$ is as yet not well established because of the rarity of this disease and the few data available on recurrence.

In cases harboring papillary thyroid microcarcinomas, after surgical resection of the ovarian tumor no further therapy involving the thyroid may be performed. In cases with larger tumors, some authors support thyroidectomy to allow for thyroglobulin monitoring and radioiodine treatment. ${ }^{12,13}$ From a histopathological point of view, thyroidectomy permits differential diagnosis between thyroid carcinoma with ovarian metastases and thyroid carcinoma in mature cystic teratoma. Moreover, thyroidectomy may be proposed, since patients with malignant SO appear to be predisposed to harboring or developing thyroid cancer and, in particular, with aggressive behavior. ${ }^{7}$ Recently, Wolff EF et al ${ }^{14}$ proposed aggressive multimodal management for malignant SO.

Malignant SO may occasionally present with a locally invasive disease and/or metastases,${ }^{15}$ which can occur in about $20 \%$ of cases. ${ }^{16}$ In a study analyzing one of the largest series of patients in the literature with malignant $\mathrm{SO}$, a mean age of 43 years was determined. ${ }^{7}$ In this study, patient survival was excellent, since only one specific-disease death was reported; $9 \%$ of patients were found to have a primary thyroid cancer and only $33 \%$ of them had a cancer confined to the thyroid gland: consequently, in this subset of patients a more invasive tendency than usually expected for differentiated thyroid cancers may be found. ${ }^{7}$ In terms of survival, Robboy et al ${ }^{17}$ performed a study on 88 patients affected by malignant $\mathrm{SO}$, which reported overall survival rates of $89 \%$ and $84 \%$ at 1 and 25 years, respectively. Marti et al ${ }^{18}$ reviewed a series of four patients from a single institution and 53 cases from the literature, comparing the extent of treatment and outcomes. After a median follow-up of 9 years, all four patients clinically remained with no evidence of disease. The pooled cumulative recurrence rate of the 57 evaluated cases was $7.5 \%$ at 25 years: on this basis, they concluded that extensive pelvic surgery and prophylactic total thyroidectomy to facilitate radioiodine therapy may be reserved for patients with gross extra-ovarian extension or distant metastases, as in our case. Like Marti et al, we consider that the latter treatment modality needs to be reserved for this set of patients, since it can impact on quality of life and fertility of affected women, given the mean young age at diagnosis.

Mean time to first tumor recurrence for papillary carcinoma in $\mathrm{SO}$ is about 4 years, though it has been reported up to 21.4 years after surgery. ${ }^{19}$ Histopatological factors predicting aggressive disease are tumor size $>10 \mathrm{~cm}$, more than $80 \%$ strumal tissue affected by carcinoma, presence of necrosis, $\geq 5$ mitoses per 10 high power field and marked cell atypia. ${ }^{19}$ Barr bodies and 46,XX karyotype have been found to play a role in disease development. ${ }^{1}$ Molecular genetics may help to differentiate benign from malignant lesions. ${ }^{14}$ Its malignant conversion has been associated with V-raf murine sarcoma viral oncogene homolog B1 (BRAF) mutations as primary thyroid carcinoma. ${ }^{11,20}$ Multiple molecular abnormalities have been reported, including point mutations in BRAF (V600E and K601E), ${ }^{14,21,22}$ in $\mathrm{HRAS}^{23}$ and NRAS, ${ }^{24}$ ret/PTC rearrangements ${ }^{25}$ and loss of heterozygosity in the PTEN region. ${ }^{26}$ Specifically in our case, no mutation was detected in exon 15 of BRAF; however, in exon 3 of NRAS, c. $182 \mathrm{~A}>\mathrm{G}$ change was detected in codon 61 (Q61R), which generates amino acid substitution: to the best of our knowledge, this is the first case in the literature harboring a change in exon 3 of N-RAS. Interestingly, frequency of NRAS mutations in primary papillary thyroid cancer, lymph node metastases and distant metastases has recently been reported in $1.2 \%, 1.3 \%$ and $14.3 \%$ of cases, respectively. ${ }^{27}$

In conclusion, we believe that our case raises some interesting considerations. First of all, pathologists should be aware of this entity and should check for the presence of point mutations suggesting an aggressive disease behavior, which could be beneficial for 
an optimal therapeutic approach: in particular, the presence of BRAF and/or NRAS point mutations may be of prognostic importance and help in the selection of candidate patients for more intensive therapeutic protocols. Secondly, in cases with non-operable metastases, management should follow that of metastatic inoperable differentiated thyroid cancer. Third, even though some authors state that the overall prognosis of malignant SO is good, or even excellent, we believe that in patients with extensive and/or aggressive disease which may impact prognosis and survival, a long-term follow-up is needed, especially in cases with large tumors and aggressive biological behavior. Fourth, in cases in which hormone withdrawal could cause progression of disease, rhTSH protocol should be preferred for radioiodine treatment. It is important to have a multidisciplinary board to evaluate these patients and, in every case, treatment should be personalized on the basis of patient and tumor characteristics. Although most of the knowledge in this field comes from case reports, efforts should be made to standardize the management of patients affected by malignant $\mathrm{SO}$, including the application of practice guidelines.

\section{REFERENCES}

1. Roth LM, Talerman A, 2007 The enigma of struma ovarii. Pathology 39: 139-146.

2. Pick L, 1903 Beitrag zur Lehre von den Greschwülsten über struma thyroidaea ovarii aberrata. Verhandlungen der Berliner Medizinischen Geselschaft 33: 139-146.

3. Rosenblum NG, LiVolsi VA, Edmonds PR, Mikuta JJ, 1989 Malignant struma ovarii. Gynecol Oncol 32: 224-227.

4. Volpi E, Ferrero A, Nasi PG, Sismondi P, 2003 Malignant struma ovarii: a case report of laparoscopic management. Gynecol Oncol 90: 191-194.

5. Dardik RB, Dardik M, Westra W, Montz FJ, 1999 Malignant struma ovarii: two case reports and a review of the literature. Gynecol Oncol 73: 447-451.

6. Yassa L, Sadow P, Marqusee E, 2008 Malignant struma ovarii. Nat Clin Pract Endocrinol Metab 4: 469-472.

7. Goffredo P, Sawka AM, Pura J, Adam MA, Roman SA, Sosa JA, 2015 Malignant struma ovarii: a populationlevel analysis of a large series of 68 patients. Thyroid 25: 211-215.

8. Yoo SC, Chang KH, Lyu MO, Chang SJ, Ryu HS, Kim HS, 2008 Clinical characteristics of struma ovarii. J Gynecol Oncol 19: 135-138.

9. Matsuda K, Maehama T, Kanazawa K, 2001 Malignant struma ovarii with thyrotoxicosis. Gynecol Oncol 82: 575-577.

10. Ciccarelli A, Valdes-Socin H, Parma J, et al, 2004 Thyrotoxic adenoma followed by atypical hyperthyroidism due to struma ovarii: clinical and genetic studies. Eur J Endocrinol 150: 431-437.

11. De Simone CP, Lele SM, Modesitt SC, 2003 Malignant struma ovarii: a case report and analysis of cases reported in the literature with focus on survival and I-131 therapy. Gynecol Oncol 89: 543-548.

12. Dane C, Ekmez M, Karaca A, Ak A, Dane B, 2012 Follicular variant of papillary thyroid carcinoma arising from a dermoid cyst: a rare malignancy in young women and review of the literature. Taiwan J Obstet Gynecol 51: 421-425.

13. Uzum AK, Iyibozkurt C, Canbaz B, et al, 2013 Management and follow-up results of an incidental thyroid carcinoma in a young woman with ovarian teratoma. Gynecol Endocrinol 29: 724-726.

14. Wolff EF, Hughes M, Merino MJ, et al, 2010 Expression of benign and malignant thyroid tissue in ovarian teratomas and the importance of multimodal management as illustrated by a BRAF-positive follicular variant of papillary thyroid cancer. Thyroid 20: 981-987.

15. Zhu Y, Wang C, Zhang G-N, et al, 2016 Papillary thyroid cancer located in malignant struma ovarii with omentum metastasis: a case report and review of the literature. World J Surg Oncol 14: 17.

16. Makani S, Kim W, Gaba AR, 2004 Struma Ovarii with a focus of papillary thyroid cancer: a case report and review of the literature. Gynecol Oncol 94: 835-839.

17. Robboy SJ, Shaco-Levy R, Peng RY, et al, 2009 Malignant struma ovarii: an analysis of 88 cases, including 27 with extraovarian spread. Int J Gynecol Pathol 28: 405-422.

18. Marti JL, Clark VE, Harper H, Chhieng DC, Sosa JA, Roman SA, 2012 Optimal surgical management of welldifferentiated thyroid cancer arising in struma ovarii: a series of 4 patients and a review of 53 reported cases. Thyroid 22: 400-406.

19. Roth LM, Miller AW 3rd, Talerman A, 2008 Typical thyroid-type carcinoma arising in struma ovarii: a report of 4 cases and review of the literature. Int J Gynecol Pathol 27: 496-506.

20. Elisei R, Romei C, Castagna MG, et al, 2005 RET/ PTC3 rearrangement and thyroid differentiation gene analysis in a struma ovarii fortuitously revealed by elevated serum thyroglobulin concentration. Thyroid 15: 1355-1361.

21. Flavin R, Smyth P, Crotty P, et al, 2007 BRAF T1799A mutation occurring in a case of malignant struma ovarii. Int J Surg Pathol 15: 116-120.

22. Schmidt J, Derr V, Heinrich MC, et al, 2007 BRAF in papillary thyroid carcinoma of ovary (struma ovarii). Am J Surg Pathol 31: 1337-1343.

23. Coyne C, Nikiforov YE, 2010 RAS mutation-positive 
follicular variant of papillary thyroid carcinoma arising in a struma ovarii. Endocr Pathol 21: 144-147.

24. Celestino R, Magalhães J, Castro P, et al, 2009 A follicular variant of papillary thyroid carcinoma in struma ovarii. Case report with unique molecular alterations. Histopathology 55: 482-487.

25. Boutross-Tadross O, Saleh R, Asa SL, 2007 Follicular variant papillary thyroid carcinoma arising in struma ovarii. Endocr Pathol 18: 182-186.
26. Tate G, Tajiri T, Suzuki T, Mitsuya T, 2009 Mutations of the KIT gene and loss of heterozygosity of the PTEN region in a primary malignant melanoma arising from a mature cystic teratoma of the ovary. Cancer Genet Cytogenet 190: 15-20.

27. Melo M, Gaspar da Rocha A, Batista R, et al, 2017 TERT, BRAF, and NRAS in Primary Thyroid Cancer and Metastatic Disease. J Clin Endocrinol Metab 102: 1898-1907. 